\title{
Clinical Significance of Composition Changes in T-cell Subpopulations After Chemotherapy in Patients With Gastric Cancer
}

\author{
MIN-KYUNG YEO ${ }^{*}$, PUREUM SUN ${ }^{2 *}$, CHAEUK CHUNG ${ }^{3}$, JAE HO PARK $^{4}$, SUN HYUNG KANG ${ }^{4}$, \\ HEE SEOK MOON ${ }^{4}$, JAE KYU SUNG ${ }^{4}$, HYUN YONG JEONG ${ }^{4}$ and JU SEOK KIM ${ }^{4}$ \\ ${ }^{1}$ Department of Pathology, Chungnam National University School of Medicine, Daejeon, Republic of Korea; \\ ${ }^{2}$ Research Institute for Medical Sciences, College of Medicine, \\ Chungnam National University, Daejeon, Republic of Korea; \\ ${ }^{3}$ Division of Pulmonology, Department of Internal Medicine, \\ College of Medicine, Chungnam National University, Daejeon, Republic of Korea; \\ ${ }^{4}$ Department of Internal Medicine, Chungnam National University School of Medicine, Daejeon, Republic of Korea
}

\begin{abstract}
Background/Aim: New therapeutic agents and prognostic biomarkers for gastric cancer are needed. We analyzed the composition of peripheral blood T-cell subpopulations in response to chemotherapy in patients with gastric cancer. Patients and Methods: Peripheral blood samples were collected from patients diagnosed with gastric cancer before and after chemotherapy (FOLFOX; oxaliplatin, 5-fluorouracil, and leucovorin). Peripheral blood mononuclear cells were isolated. Patients were divided into responder $(n=5)$ and non-responder groups $(n=2)$ based on their chemotherapy outcomes. Results: Non-responders showed lower numbers of CD4+/total cells and CD8+/total cells after chemotherapy compared to the responder group, but the difference was not significant ( $p=0.905, p=0.095)$. Nä̈ve T, central memory $T$, effector memory $T$ and effector T-cell counts differed in both groups after chemotherapy. Conclusion: Changes in peripheral T-cell subpopulations after chemotherapy were confirmed in patients with gastric cancer, which may be a prognostic predictor and development of therapeutic agents.
\end{abstract}

This article is freely accessible online.

*These Authors contributed equally to this study.

Correspondence to: Ju Seok Kim, MD, Ph.D., Department of Internal Medicine, Chungnam National University School of Medicine, 282 Munhwa-ro, Jung-gu, Daejeon 35015, Republic of Korea. Tel: +82 449953420, Fax: +82 449954252, e-mail: showsik@hanmail.net

Key Words: Peripheral T-cell subpopulation, gastric cancer, immunotherapy, prognosis.
Gastric cancer is the fifth most frequently diagnosed cancer and third most frequent cause of cancer-related death worldwide (1). Early-stage gastric cancer has a high 5-year survival rate of more than $90 \%$; however, lymph node metastasis and disease recurrence are common in the advanced stage after treatment, resulting in poor prognosis (2). Various therapies such as radiation therapy or adjuvant chemotherapy have been administered to patients with gastric cancer after surgery to improve prognosis. However, these therapies do not reduce recurrence or increase the survival rate of patients; therefore, they are no longer recommended for improving prognosis in patients with gastric cancer $(3,4)$. Palliative chemotherapy is administered to patients when surgery is impossible and the treatment outcome is non-significant because of distant metastasis and poor prognosis $(5,6)$.

Programmed death-ligand 1 (PD-L1) is a protein present on the surface of cancer cells or hematopoietic cells, whereas programmed cell death protein 1 (PD1) is a protein present on the surface of T-cells. When PD-L1 binds to PD1, T-cells cannot attack cancer cells (7). Recently, anti-PD1 and antiPD-L1 agents that prevent the binding of these two proteins have replaced conventional cytotoxic agents $(8,9)$. Immunotherapy has almost doubled the overall median survival rates of patients with melanoma and non-small cell lung cancer, and is being used for other cancer types $(10,11)$. Biomarkers play an important role in objectively measuring and evaluating the response of drugs to normal biological processes, disease progression, and treatment methods, and have clinical value when they can be easily identified such as in peripheral blood samples (12). In patients with cancer, understanding the difference between responders and nonresponders to specific drugs or monitoring treatment effects 
in vivo $35: 2417-2424(2021)$

Table I. Patient characteristics.

\begin{tabular}{|c|c|c|c|c|c|}
\hline & Age & Gender & Stage & Initial chemotherapy regimen & Response to chemotherapy \\
\hline 1 & 71 & Male & IV & FOLFOX & Progressive disease \\
\hline 2 & 68 & Male & IV & FOLFOX & Progressive disease \\
\hline 3 & 69 & Female & IV & FOLFOX & Partial response \\
\hline 4 & 76 & Male & IV & FOLFOX & Stable disease \\
\hline 5 & 63 & Male & IV & FOLFOX & Stable disease \\
\hline 6 & 49 & Male & IV & FOLFOX & Stable disease \\
\hline 7 & 55 & Male & IV & FOLFOX & Stable disease \\
\hline
\end{tabular}

FOLFOX: oxaliplatin, 5-fluorouracil, leucovorin.

helps improve prognosis (13). Anti-PD1 agents and anti-PDL1 agents also cause increased expression of PD1 or PD-L1 as a therapeutic indication; in such cases, the therapeutic effect is generally good $(14,15)$.

Gastric cancer cells generally exhibit high diversity in their structures, making it difficult to develop new therapeutic agents or biomarkers; additionally, conventional cytotoxic agents result in poor clinical outcomes (16). Because of these diverse characteristics, administering immunotherapeutic agents for treating gastric cancer is also difficult, and thus studies of new therapeutic agents and biomarkers is necessary $(17,18)$.

Therefore, this study was conducted to analyze the characteristics of the peripheral blood T-cell subpopulation in patients with gastric cancer before and after chemotherapy to determine the association between the composition of peripheral T-cell subpopulations and gastric cancer prognosis. We investigated the use of the peripheral blood T-cell subpopulation as a biomarker for gastric cancer prognosis and possibility of using immunotherapy for treating this cancer.

\section{Patients and Methods}

Study design and patient selection. Patients over 18 years of age who were histologically diagnosed with gastric cancer at the Chungnam National University Hospital (Daejeon, Republic of Korea) between September 2019 and March 2020 and who had a World Health Organization performance status of 0 or 1 were enrolled. Those with a history of previous chemotherapy, secondary malignancy, and inadequate renal function (serum creatinine clearance $\leq 60 \mathrm{ml} / \mathrm{min}$ ) were excluded. Pre-treatment clinical evaluation included determination of the complete blood cell count with differential and serum multichannel chemical analysis. For clinical staging, upper gastrointestinal endoscopy with biopsy, abdominal computed tomography, and chest radiography were performed. This study was approved by the institutional review board of the Chungnam National University Hospital (2019-09-047).

Chemotherapy and response assessment. The chemotherapy regimen for all patients was the same (FOLFOX; oxaliplatin, 5-fluorouracil, and leucovorin) and was administered at intervals of 2 weeks. Tumor response or safety aspects were considered while adjusting the dose and interval of the anticancer drug. Peripheral blood was collected from all patients and analyzed before and after 3 cycles of chemotherapy. Before each cycle, complete blood count and levels of electrolyte and serum creatinine were analyzed, and a liver function test was performed. A reduction in the primary tumor size was measured as a response to chemotherapy by endoscopy and abdominal computed tomography (CT). Complete disappearance of lesions after endoscopy and CT was considered as a clinically complete response (CR). A tumor size reduction greater than $50 \%$ compared with the initial tumor size was defined as a partial response (PR). A minor response or no change after chemotherapy was considered as stable disease (SD). New lesions or a greater than $25 \%$ increase in primary tumor size were considered as progressive disease (PD). Patients showing $\mathrm{CR}, \mathrm{PR}$, and $\mathrm{SD}$ were considered as responders, whereas those showing PD were considered as non-responders.

Flow cytometry and intracellular cytokine staining. The following human antibodies were used: anti-PD-1 (FITC), anti-CD4 (PerCPCy5.5), anti-CD127 (APC), anti-CD8 (AF700), and anti-CCR7 (BV786) from BioLegend (San Diego, CA, USA). Anti-CD45RA (PE-Cy7), anti-CD3 (APC-Cy7), and anti-CD25 (BV421) were purchased from BD Biosciences (San Jose, CA, USA). To exclude dead cells from the analysis, the viability dye LIVE/DEAD ${ }^{\circledR}$ Fixable Aqua (Thermo Fisher Scientific, Waltham, MA, USA) was used as a live/dead marker. For intracellular staining of forkhead box protein P3, the cells were fixed and permeabilized using the Foxp3/Transcription factor staining buffer set (eBioscience, San Diego, CA, USA) and incubated with an antibody against the T-reg transcription factor marker for $30 \mathrm{~min}$ at $4^{\circ} \mathrm{C}$. After washing, the stained cells were analyzed using BD LSR Fortessa X-20 (BD Biosciences) and FlowJo software (TreeStar, Ashland, OR, USA).

Preparation of cryopreserved human peripheral blood mononuclear cells (PBMCs). PBMCs were separated from peripheral blood using lymphocyte separation medium (Corning, Inc., Corning, NY, USA) and by gradient centrifugation, and all samples were cryopreserved in Mr. Frosty ${ }^{\mathrm{TM}}$ Freezing Container (Thermo Fisher Scientific) at $-80^{\circ} \mathrm{C}$. The samples were then transferred to liquid nitrogen until further use. Cryopreserved PBMCs were rapidly thawed and rested in Roswell Park Memorial Institute 1640 medium supplemented with $10 \%$ fetal bovine serum and $1 \%$ penicillin/streptomycin (all from Corning, Inc.) and incubated for $15 \mathrm{~h}$ at $37^{\circ} \mathrm{C}$ in a humidified atmosphere with $5 \% \mathrm{CO}_{2}$.

Statistical analysis. Changes in PBMCs of each patient before and after chemotherapy were analyzed using the Mann-Whitney $U$ and 

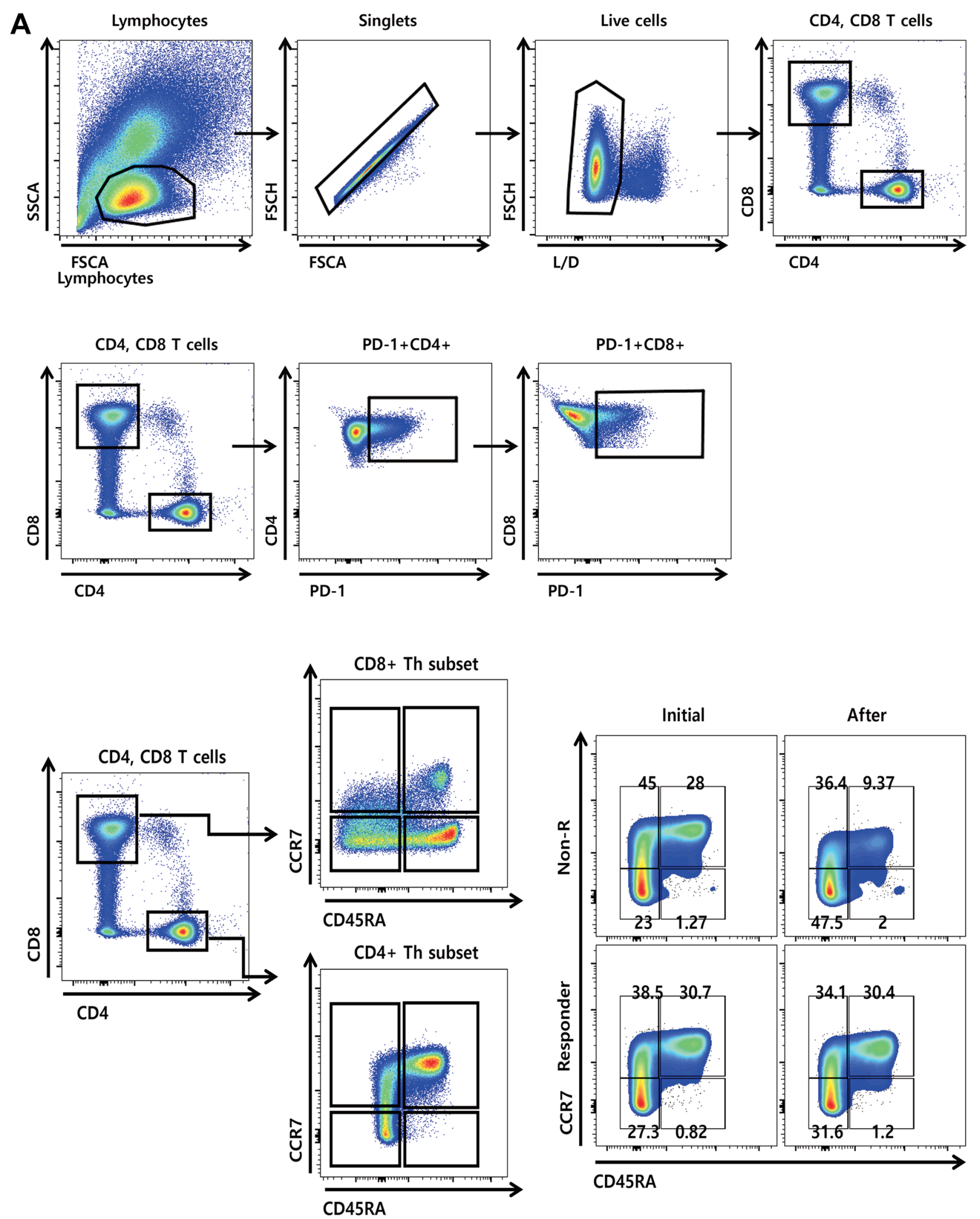

Figure 1. Continued 
B
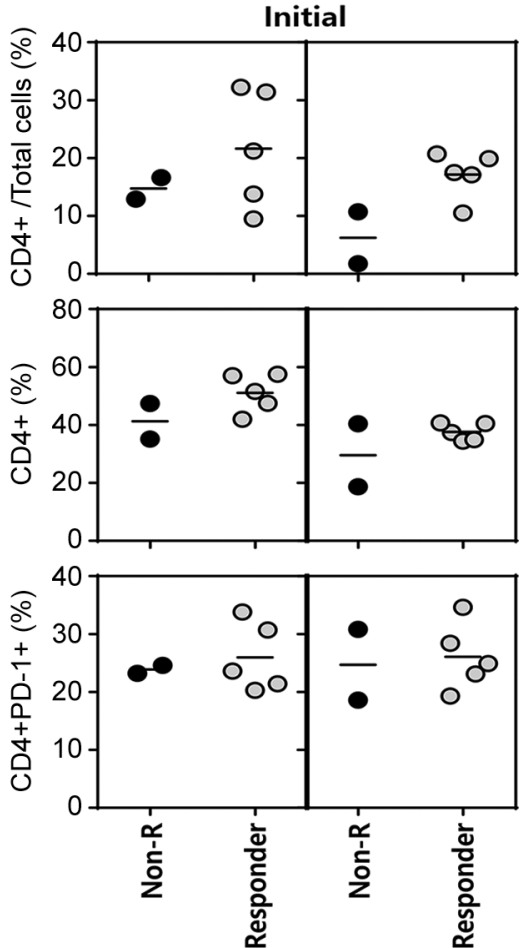
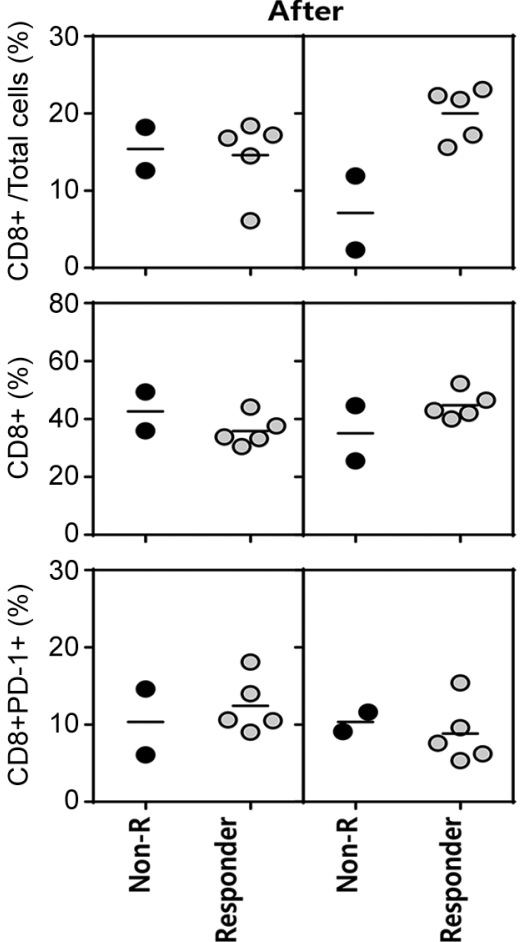

Figure 1. Peripheral T-cell subpopulations. (A) Gating strategy to define T-cell subpopulations in PBMCs. (B) CD4+and CD8+T-cell subpopulations and PDI+ cell in PBMCs were compared in the responder and non-responder groups. Non-R: Non-responder; PBMCs: peripheral blood mononuclear cells.

Wilcoxon tests. All analyses were performed using SPSS software (version 19.0, SPSS, Inc., Chicago, IL, USA). $p$-Values were determined using two-sided tests and values $<0.05$ were considered as significant.

\section{Results}

Patient characteristics. Seven patients who were diagnosed with gastric cancer and progressed to response evaluation after 3 cycles of chemotherapy were enrolled in the study; the clinical characteristics of these patients are summarized in Table I. The results of response evaluation showed that two patients were non-responders and five were responders (CR: 0, PR: 1, SD: 4, and PD: 2). Among all patients, 86\% $(n=6)$ were male and their mean age was $64.42(9.48)$ years. All patients were in stage IV, and the initial chemotherapy regimen was the same as FOLFOX.

Peripheral T-cell subpopulations. The gating strategy used to define T-cell subpopulations in PBMCs is summarized in (Figure 1A). CD4+/total cell (\%), CD8+/total cell (\%), CD4+ (\%), CD8+ (\%), CD4+PD-1 (\%), and CD8+PD-1 (\%) of patients in both the groups were compared (Figure 1B). In the non-responder group, the number of CD4+/total cells (\%) and CD8+/total cells (\%) decreased after chemotherapy compared to in the responder group but the difference was not significant $(p=0.905$ and $p=0.095)$. However, CD4+ $(\%)$, CD8+ (\%), CD4+PD-1 (\%), and CD8+PD-1 (\%) showed no difference before and after treatment in both groups.

Composition of T-Cell subsets change after chemotherapy. Compositional changes in naïve T-cells (TN), central memory T-cells (TCM), effector memory T-cells (TEM), and effector T-cells (TEFF) before and after chemotherapy in both groups were compared (Figure 2A). The number of CD4+ and CD8+ T-cell subsets after chemotherapy in the two groups differed, but the difference was not significant. Each T-cell subset was further categorized as TN/CD4+, TN/CD8+; TCM/CD4+, TCM/CD8+; TEM/CD4+, TEM/CD8+, and TEFF/CD4+ and TEFF/CD8+ groups to analyze the changes after chemotherapy (Figure 2B). However, the differences between the number of subsets in both the groups were not significant.

TCM/TEFF ratio differed between groups after chemotherapy. In both groups, the $\mathrm{CD} 4+\mathrm{TCM} / \mathrm{TEFF}$ ratio was similar before and after chemotherapy, whereas the CD8+ TCM/TEFF ratio decreased after chemotherapy 

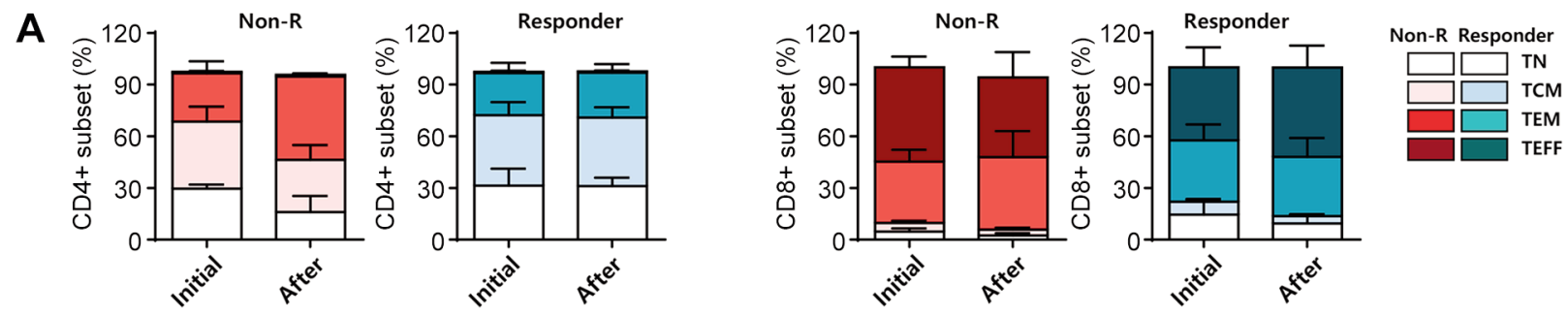

B
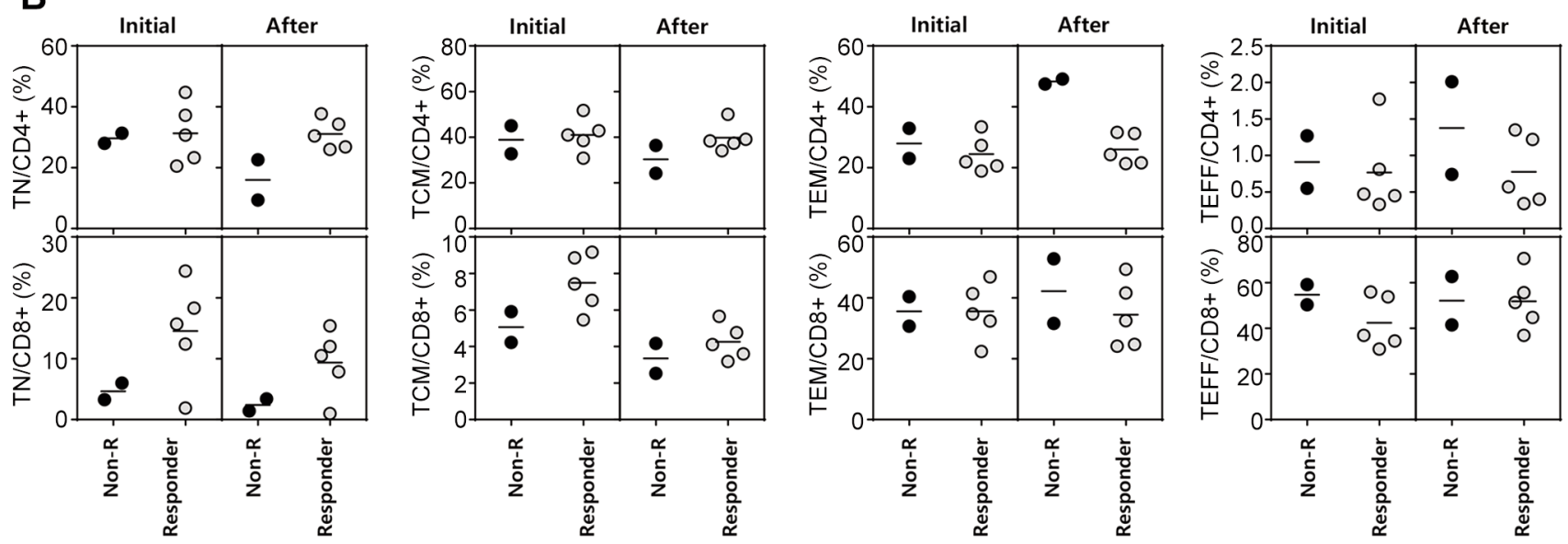

Figure 2. Changes of T-cell subsets after chemotherapy. (A) Changes in CD4+ and CD8+T-cell subsets after chemotherapy in responder and nonresponder groups. (B) Changes in TN, TCN, TEM, and TEFF ratio after chemotherapy in responder and non-responder groups. TN: Nä̈ve T-cell; TCM: central memory T-cell; TEM: effector memory T-cell; TEFF: effector T-cell; Non-R: non-responder.

CD4+ TCM/TEFF ratio

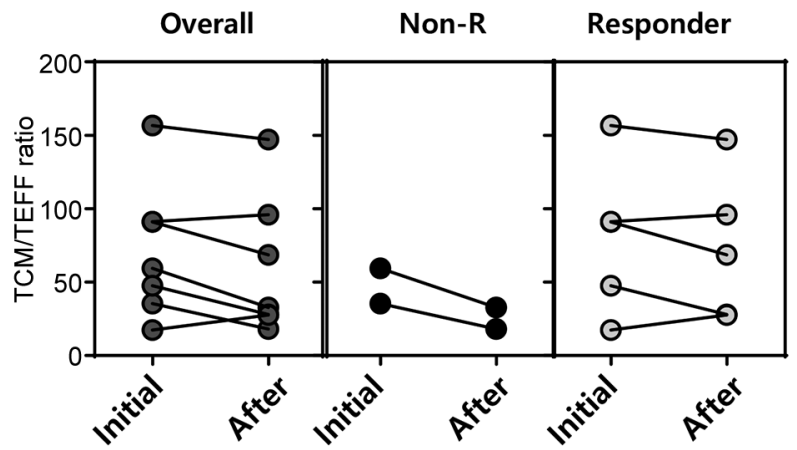

CD8+ TCM/TEFF ratio

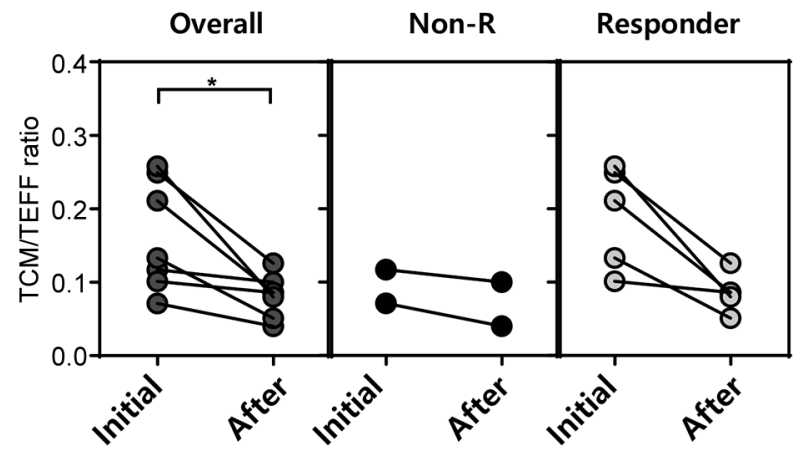

Figure 3. Changes in TCM/TEFF ratio after chemotherapy in responder and non-responder groups. TCM: Central memory T-cell; TEFF: effector $T$-cell; Non-R: non-responder.

(Figure 3); however, the differences were not significant $(p=0.109$ and $p=0.156)$. The CD4+ TCM/TEFF and CD8+ $\mathrm{TCM} / \mathrm{TEFF}$ ratios in both groups differed after chemotherapy but the differences were not significant $(p=0.437$ and $p=0.062)$.

Changes in regulatory $T$-cell chemotherapy. To determine the role of regulatory T-cells (T-reg), changes in the number of
PD1+/Fr. 1, PD1+/Fr. L1, and PD1+/Fr. 111 (\%) in both groups were analyzed after chemotherapy (Figure 4A). In the responder group, the number of PD1+/Fr. 1, PD1+/Fr. L1, and PD1+/Fr. 111 (\%) did not change after chemotherapy, whereas in the nonresponder group, the number of PD1+/Fr. I and PD1+/Fr. $11(\%)$ decreased after chemotherapy but the difference was not significant ( $p=0.238$ and $p=0.095)$. No significant difference was observed between the number of Fr. 1/CD25+CD127-/or 
A

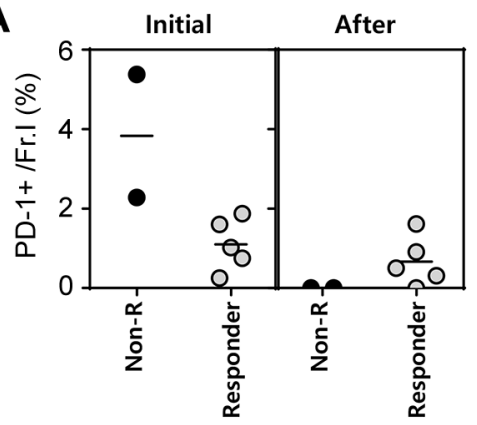

B

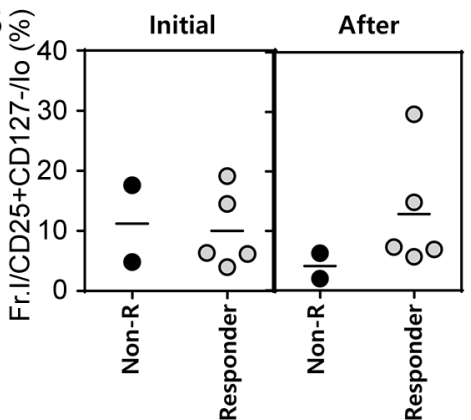

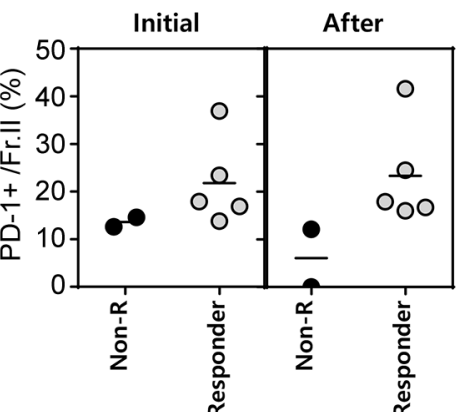

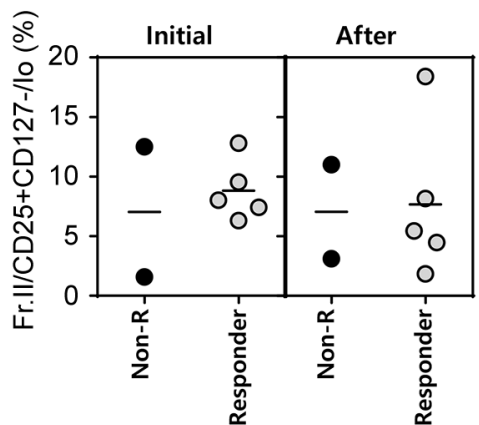

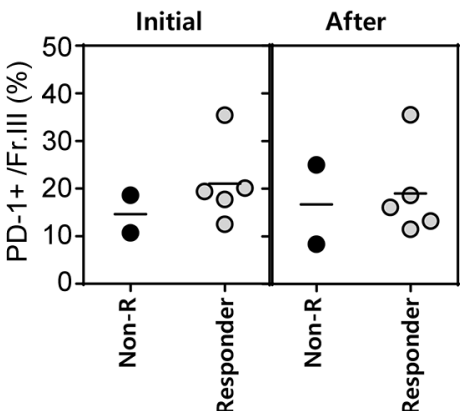

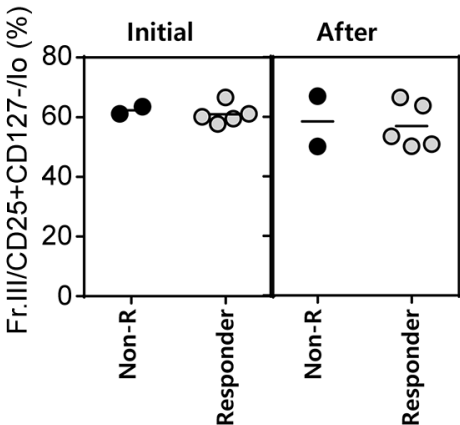

Figure 4. Changes of T-cell subsets after chemotherapy. (A) Changes in PD1+/Fr. l, PD1+/Fr. ll, and PD1+/Fr. lll (\%) after chemotherapy in responder and non-responder groups. (B) Changes in Fr. l/CD25+CD127-/or low cells, Fr. ll/CD25+CD127-/or low cells, and Fr. lll/CD25+CD127lor low cells after chemotherapy in responder and non-responder groups. Non-R: Non-responder.

low cells, Fr. 11/CD25+CD127-/or low cells, and Fr. 111/CD25+CD127-/or low cells (Figure 4B) $(p=0.238, p=0.381$, and $p=0.667$ ) in the two groups after chemotherapy.

\section{Discussion}

In this study, we compared the compositional changes in peripheral T-cell subpopulations in responder and nonresponder groups of patients with gastric cancer. We observed compositional changes in the peripheral T-cell subpopulations; however, as the number of enrolled patients in this study was small, significance could not be determined. However, to the best of our knowledge, this is the first study to analyze the compositional changes in peripheral T-cell subpopulations in patients with gastric cancer and clinical outcomes after chemotherapy. New treatment agents are required to treat gastric cancer, as the efficacy of existing cytotoxic agents is low $(17,18)$. The failure to maintain homeostasis between immune activation and immune suppression plays an important role in cancer development (19). The immune system can act to either induce or prevent cancer, but the role of T-cells is also important (20), as CD8+ T-cells remove tumor cells during cancer development $(21,22)$. Previous studies showed that the population of CD8+ T-cells is significantly reduced in patients with head and neck cancer (23). Changes in the composition of T-cell subpopulations are associated with cancer incidence; however, in this study, we found that changes in the composition of CD4+ cells and CD8+ cells were associated with the prognosis of gastric cancer, although the difference was not significant. In a normal host, T-cell memory lasts for a lifetime because some memory T-cells self-renew following exposure to the same antigen (24). CD4+ and CD8+ TEM produce effector cytokines, express perforin, and exert cytolytic activity. The self-renewing state required to be maintained by these signals requires a microenvironment of lymphoid organs (25). When antigens are removed, most effector cells are eliminated, whereas the self-renewing population is retained (26). CD4+ and CD8+ memory T-cells are correlated with the clinical response in melanoma patients treated with ipilimumab (27). In this study, changes in TN, TCN, TEM, and TEFF after initial chemotherapy were analyzed, and alterations in their composition before and after chemotherapy were observed in both groups. In 22 patients with non-small cell lung cancer treated with nivolumab, progression-free survival was significantly high with a high TCM/TEFF ratio (median survival: 91 vs. 215 days) (28). In the present study, CD4+ TCM/TEFF and CD8+ TCM/TEFF ratios did not significantly differ between groups after chemotherapy. Changes in the Tcell composition are considered to be related to the prognosis of gastric cancer, and further studies are required to confirm this hypothesis. 
T-regs are characterized by co-expression of CD4 and $\mathrm{CD} 25$, and their numbers are increased in lung, breast, and ovarian cancers $(29,30)$. T-regs can be classified as Fr. 1, Fr. 11 , and Fr. depending on the expression of CD4 and CD25 (31). To confirm the changes in the number of T-regs, Fr. 1, Fr. 1l, and Fr. Lll were compared between groups before and after chemotherapy. In the non-responder group, PD1+/Fr. L and PD1+/Fr. 11 (\%) were decreased after chemotherapy $(p=0.238, p=0.095)$. Because the number of patients analyzed was small, significant results were not observed.

Changes in peripheral T-cell subpopulations were identified in patients with gastric cancer, and analysis was performed by considering chemotherapy as an important aspect. One limitation of this study is that the results are difficult to generalize because of the small sample size of patients and the mechanism involved in changes in T-cell subpopulations was not determined.

In conclusion, the composition of peripheral T-cell subpopulations was changed in patients with gastric cancer after chemotherapy. Although there was no statistical significance due to the small number of enrolled patients, it is meaningful that changes in T-cell subpopulations were confirmed. Therefore, the possibility of an association between the composition of peripheral T-cell subpopulations and gastric cancer prognosis is actual. Peripheral T-cell subpopulations may be useful as biomarkers for gastric cancer prognosis and development of therapeutic agents. However, further research on this is still needed.

\section{Conflicts of Interest}

The Authors declare that there are no conflicts of interest associated with the work presented in this manuscript.

\section{Authors' Contributions}

Conceptualization, M.K.Y. and J.S.K.; Funding acquisition, J.S.K.; Investigation, P.S., C.C., J.H.P., and S.H.K.; Methodology, H.S.M. and M.K.Y.; Supervision, H.Y.J.; Validation, Writing original draft, P.S., C.C. and J.H.P.; Writing-review \& editing, M.K.Y., and J.S.K.

\section{Acknowledgements}

This work was supported by the research fund of Chungnam National University.

\section{References}

1 Ramazani Y, Mardani E, Najafi F, Moradinazar M and Amini M: Epidemiology of gastric cancer in North Africa and the Middle East from 1990 to 2017. J Gastrointest Cancer, 2020. PMID: 33051794. DOI: 10.1007/s12029-020-00533-6

2 Nashimoto A, Nakajima T, Furukawa H, Kitamura M, Kinoshita T, Yamamura Y, Sasako M, Kunii Y, Motohashi H, Yamamoto S and Gastric Cancer Surgical Study Group, Japan Clinical
Oncology Group: Randomized trial of adjuvant chemotherapy with mitomycin, Fluorouracil, and Cytosine arabinoside followed by oral Fluorouracil in serosa-negative gastric cancer: Japan Clinical Oncology Group 9206-1. J Clin Oncol 21(12): 22822287, 2003. PMID: 12805327. DOI: 10.1200/JCO.2003.06.103

3 Ronellenfitsch U, Schwarzbach M, Hofheinz R, Kienle P, Kieser M, Slanger TE, Burmeister B, Kelsen D, Niedzwiecki D, Schuhmacher C, Urba S, van de Velde C, Walsh TN, Ychou M and Jensen K: Preoperative chemo(radio)therapy versus primary surgery for gastroesophageal adenocarcinoma: systematic review with meta-analysis combining individual patient and aggregate data. Eur J Cancer 49(15): 3149-3158, 2013. PMID: 23800671. DOI: $10.1016 /$ j.ejca.2013.05.029

4 Ajani JA, Komaki R, Putnam JB, Walsh G, Nesbitt J, Pisters PW, Lynch PM, Vaporciyan A, Smythe R, Lahoti S, Raijman I, Swisher S, Martin FD and Roth JA: A three-step strategy of induction chemotherapy then chemoradiation followed by surgery in patients with potentially resectable carcinoma of the esophagus or gastroesophageal junction. Cancer 92(2): 279-286, 2001. PMID: 11466680. DOI: 10.1002/10970142(20010715)92:2<279::aid-cncr1320>3.0.co;2-2

5 Liang X, Zhu J, Li Y, Xu Y, Chen K, Lv L and Mao W: Treatment strategies for metastatic gastric cancer: chemotherapy, palliative surgery or radiotherapy? Future Oncol 16(5): 91-102, 2020. PMID: 31868545. DOI: 10.2217/fon-2019-0495

6 Beom SH, Choi YY, Baek SE, Li SX, Lim JS, Son T, Kim HI, Cheong JH, Hyung WJ, Choi SH, Jung M, Kim HS, Jeung HC, Chung HC, Rha SY and Noh SH: Multidisciplinary treatment for patients with stage IV gastric cancer: the role of conversion surgery following chemotherapy. BMC Cancer 18(1): 1116, 2018. PMID: 30442107. DOI: 10.1186/s12885-018-4998-x

7 Hamanishi J, Mandai M, Matsumura N, Abiko K, Baba T and Konishi I: PD-1/PD-L1 blockade in cancer treatment: perspectives and issues. Int J Clin Oncol 21(3): 462-473, 2016. PMID: 26899259. DOI: 10.1007/s10147-016-0959-z

8 Gong J, Chehrazi-Raffle A, Reddi S and Salgia R: Development of PD-1 and PD-L1 inhibitors as a form of cancer immunotherapy: a comprehensive review of registration trials and future considerations. J Immunother Cancer 6(1): 8, 2018. PMID: 29357948. DOI: 10.1186/s40425-018-0316-Z

9 Park R, Williamson S, Kasi A and Saeed A: Immune therapeutics in the treatment of advanced gastric and esophageal cancer. Anticancer Res 38(10): 5569-5580, 2018. PMID: 30275174. DOI: 10.21873 /anticanres.12891

10 Hodi FS, Chesney J, Pavlick AC, Robert C, Grossmann KF, McDermott DF, Linette GP, Meyer N, Giguere JK, Agarwala SS, Shaheen M, Ernstoff MS, Minor DR, Salama AK, Taylor MH, Ott PA, Horak C, Gagnier P, Jiang J, Wolchok JD and Postow MA: Combined nivolumab and ipilimumab versus ipilimumab alone in patients with advanced melanoma: 2-year overall survival outcomes in a multicentre, randomised, controlled, phase 2 trial. Lancet Oncol 17(11): 1558-1568, 2016. PMID: 27622997. DOI: 10.1016/S1470-2045(16)30366-7

11 Hui R, Garon EB, Goldman JW, Leighl NB, Hellmann MD, Patnaik A, Gandhi L, Eder JP, Ahn MJ, Horn L, Felip E, Carcereny E, Rangwala R, Lubiniecki GM, Zhang J, Emancipator K, Roach C and Rizvi NA: Pembrolizumab as firstline therapy for patients with PD-L1-positive advanced nonsmall cell lung cancer: a phase 1 trial. Ann Oncol 28(4): 874881, 2017. PMID: 28168303. DOI: 10.1093/annonc/mdx008 
12 Best MG, Wesseling $\mathrm{P}$ and Wurdinger T: Tumor-educated platelets as a noninvasive biomarker source for cancer detection and progression monitoring. Cancer Res 78(13): 3407-3412, 2018. PMID: 29921699. DOI: 10.1158/0008-5472.CAN-18-0887

13 Taniguchi N and Kizuka Y: Glycans and cancer: role of Nglycans in cancer biomarker, progression and metastasis, and therapeutics. Adv Cancer Res 126: 11-51, 2015. PMID: 25727145. DOI: $10.1016 /$ bs.acr.2014.11.001

14 Loo K and Daud A: Emerging biomarkers as predictors to antiPD1/PD-L1 therapies in advanced melanoma. Immunotherapy 8(7): 775-784, 2016. PMID: 27349977. DOI: 10.2217/imt-20160039

15 Banna GL, Cantale O, Bersanelli M, Del Re M, Friedlaender A, Cortellini A and Addeo A: Are anti-PD1 and anti-PD-L1 alike? The non-small-cell lung cancer paradigm. Oncol Rev 14(2): 490, 2020. PMID: 32782728. DOI: 10.4081/oncol.2020.490

16 Ho SWT and Tan P: Dissection of gastric cancer heterogeneity for precision oncology. Cancer Sci 110(11): 3405-3414, 2019. PMID: 31495054. DOI: 10.1111/cas.14191

17 Coutzac C, Pernot S, Chaput N and Zaanan A: Immunotherapy in advanced gastric cancer, is it the future? Crit Rev Oncol Hematol 133: 25-32, 2019. PMID: 30661655. DOI: 10.1016/j.critrevonc.2018.10.007

18 Matsueda S and Graham DY: Immunotherapy in gastric cancer. World J Gastroenterol 20(7): 1657-1666, 2014. PMID: 24587645. DOI: 10.3748/wjg.v20.i7.1657

19 Nicolás-Ávila JÁ, Adrover JM and Hidalgo A: Neutrophils in homeostasis, immunity, and cancer. Immunity 46(1): 15-28, 2017. PMID: 28099862. DOI: 10.1016/j.immuni.2016.12.012

20 Parkin J and Cohen B: An overview of the immune system. Lancet 357(9270): 1777-1789, 2001. PMID: 11403834. DOI: 10.1016/S0140-6736(00)04904-7

21 Sato E, Olson SH, Ahn J, Bundy B, Nishikawa H, Qian F, Jungbluth AA, Frosina D, Gnjatic S, Ambrosone C, Kepner J, Odunsi T, Ritter G, Lele S, Chen YT, Ohtani H, Old LJ and Odunsi K: Intraepithelial CD8+ tumor-infiltrating lymphocytes and a high $\mathrm{CD} 8+/$ regulatory $\mathrm{T}$ cell ratio are associated with favorable prognosis in ovarian cancer. Proc Natl Acad Sci U.S.A. 102(51): 18538-18543, 2005. PMID: 16344461. DOI: 10.1073/pnas.0509182102

22 Zhang YL, Li J, Mo HY, Qiu F, Zheng LM, Qian CN and Zeng YX: Different subsets of tumor infiltrating lymphocytes correlate with NPC progression in different ways. Mol Cancer 9: 4, 2010. PMID: 20064222. DOI: 10.1186/1476-4598-9-4

23 Kuss I, Hathaway B, Ferris RL, Gooding W and Whiteside TL: Decreased absolute counts of T lymphocyte subsets and their relation to disease in squamous cell carcinoma of the head and neck. Clin Cancer Res 10(11): 3755-3762, 2004. PMID: 15173082. DOI: 10.1158/1078-0432.CCR-04-0054

24 Berger C, Jensen MC, Lansdorp PM, Gough M, Elliott C and Riddell SR: Adoptive transfer of effector CD8+ T cells derived from central memory cells establishes persistent $\mathrm{T}$ cell memory in primates. J Clin Invest 118(1): 294-305, 2008. PMID: 18060041. DOI: $10.1172 / \mathrm{JCI} 32103$
25 Fearon DT, Manders P and Wagner SD: Arrested differentiation, the self-renewing memory lymphocyte, and vaccination. Science 293(5528): 248-250, 2001. PMID: 11452114. DOI: $10.1126 /$ science. 1062589

26 Gattinoni L, Lugli E, Ji Y, Pos Z, Paulos CM, Quigley MF, Almeida JR, Gostick E, Yu Z, Carpenito C, Wang E, Douek DC, Price DA, June CH, Marincola FM, Roederer M and Restifo NP: A human memory $\mathrm{T}$ cell subset with stem cell-like properties. Nat Med 17(10): 1290-1297, 2011. PMID: 21926977. DOI: $10.1038 / \mathrm{nm} .2446$

27 Tietze JK, Angelova D, Heppt MV, Reinholz M, Murphy WJ, Spannagl M, Ruzicka $\mathrm{T}$ and Berking C: The proportion of circulating $\mathrm{CD} 45 \mathrm{RO}^{+} \mathrm{CD} 8{ }^{+}$memory $\mathrm{T}$ cells is correlated with clinical response in melanoma patients treated with ipilimumab. Eur J Cancer 75: 268-279, 2017. PMID: 28242504. DOI: 10.1016/j.ejca.2016.12.031

28 Manjarrez-Orduño N, Menard LC, Kansal S, Fischer P, Kakrecha B, Jiang C, Cunningham M, Greenawalt D, Patel V, Yang M, Golhar R, Carman JA, Lezhnin S, Dai H, Kayne PS, Suchard SJ, Bernstein SH and Nadler SG: Circulating T cell subpopulations correlate with immune responses at the tumor site and clinical response to PD1 inhibition in non-small cell lung cancer. Front Immunol 9: 1613, 2018. PMID: 30123214. DOI: $10.3389 /$ fimmu.2018.01613

29 Woo EY, Chu CS, Goletz TJ, Schlienger K, Yeh H, Coukos G, Rubin SC, Kaiser LR and June CH: Regulatory CD4(+) CD25(+) T cells in tumors from patients with early-stage nonsmall cell lung cancer and late-stage ovarian cancer. Cancer Res 61(12): 4766-4772, 2001. PMID: 11406550.

30 Liyanage UK, Moore TT, Joo HG, Tanaka Y, Herrmann V, Doherty G, Drebin JA, Strasberg SM, Eberlein TJ, Goedegebuure PS and Linehan DC: Prevalence of regulatory T cells is increased in peripheral blood and tumor microenvironment of patients with pancreas or breast adenocarcinoma. J Immunol 169(5): 2756-2761, 2002. PMID: 12193750. DOI: 10.4049/jimmunol.169.5.2756

31 Cha Z, Gu H, Zang Y, Wang Z, Li J, Huang W, Qin A, Zhu L, Tu X, Cheng N, Song H and Qian B: The prevalence and function of $\mathrm{CD} 4{ }^{+} \mathrm{CXCR} 5+\mathrm{Foxp} 3+$ follicular regulatory $\mathrm{T}$ cells in diffuse large B cell lymphoma. Int Immunopharmacol 61: 132139, 2018. PMID: 29870918. DOI: 10.1016/j.intimp. 2018.05 .025
Received March 16, 2021

Revised April 1, 2021

Accepted April 13, 2021 